\title{
Atividade física de lazer e saúde: uma revisão sistemática
}

\author{
Cintbia Lopes da Silva* \\ Marcio Ferreira de Souza** \\ Silvio Rossi Filho*** \\ Luciene Ferreira da Silva**** \\ Ana Carolina Capellini Rigoni*****
}

\begin{abstract}
Resumo
Neste trabalho analisou-se o que foi produzido na literatura científica sobre atividade física de lazer e saúde nos últimos seis anos (2010-2015). Há discussões sobre o tema que envolvem tanto conhecimentos das ciências biológicas como das humanas e para uma intervenção social efetiva é fundamental que se identifiquem tais conhecimentos. Para isso, foi realizada uma revisão sistemática da literatura e utilizou-se como referência as bases de dados Lilacs, Scielo e os artigos publicados na Revista Brasileira de Atividade Física \& Saúde. Observou-se que a relação entre atividade física de lazer e saúde, na maioria dos estudos, é compreendida como forma de minimizar ou evitar doenças. Nos artigos selecionados, o lazer é categoria relacionada ao tempo e local em que os sujeitos realizam a atividade física. Concluiu-se que há pouca exploração das contribuições dos estudos do lazer para a saúde da população.

Palavras-chave: Atividades de Lazer, Assistência à Saúde, Promoção da Saúde.
\end{abstract}

\section{Leisure physical activity and health: a systematic review}

\begin{abstract}
This paper analyzes what has been produced in the scientific literature on leisure and health physical activity in the last six years (2010-2015). There are discussions on the subject that involve both biological and human sciences knowledge and for effective social intervention it is essential that such knowledge be identified. For this, a systematic review of the literature was carried out and the Lilacs, Scielo and articles published in the Brazilian Journal of Physical Activity \& Health were used as reference. It was observed that the relation between physical activity of leisure and health, in most studies, is understood as a way of minimizing or avoiding diseases. In selected articles, leisure is a category related to the time and place in which subjects perform physical activity. It was concluded that there is little exploration of the contributions of leisure studies to the health of the population. Keywords: Leisure Activities, Health Care Assistance, Health Promotion.
\end{abstract}

\footnotetext{
* Doutora e Mestre pela UNICAMP. Pós-doutorado pela Faculdade de Motricidade Humana da Universidade Técnica de Lisboa. Professora e pesquisadora da Universidade Metodista de Piracicaba no Programa de Pós-graduação em Ciências do Movimento Humano e nos cursos de graduação em Educação Física - Bacharelado e Licenciatura.

** Doutorando em Ciências do Movimento Humano pela Universidade Metodista de Piracicaba.

*** Mestrando em Ciências do Movimento Humano pela Universidade Metodista de Piracicaba.

**** Professora do departamento de educação da UNESP-Bauru

***** Doutora e Mestre pela UNICAMP. Professora do Programa de Pós-Graduação em Ciências do Movimento Humano da Universidade Metodista de Piracicaba.
} 


\section{Introdução}

A atividade física é definida, segundo Fraga (2012), com base no Glossário Temático de Promoção da Saúde do Ministério da Saúde (Ministério da Saúde, 2012), como toda movimentação corporal que implica em qualquer gasto de energia acima dos valores considerados como "estado de repouso". Isso pode envolver atividades como correr, subir escadas, andar, limpar a casa, etc. Por outro lado, o termo quando está associado ao lazer compreende as atividades realizadas no tempo disponível das obrigações sociais ou, ainda, como sinônimo dos conteúdos físicos e esportivos do lazer, relacionado a atividades como esportes, ginásticas, passeios, pesca etc. (Isayama, 2007).

A saúde, como categoria associada a atividade física de lazer, pode indicar a questão do direito dos sujeitos a vivência de atividades de livre escolha, com interesse único e exclusivo na atividade em si. A noção de saúde, nesse caso, seria algo próximo a afirmação de Carvalho (2001, pp.14):

A saúde resulta de possibilidades, que abrangem as condições de vida, de modo geral, e, em particular, ter acesso a trabalho, serviços de saúde, moradia, alimentação, lazer conquistados - por direito ou por interesse - ao longo da vida. Tem saúde quem tem condições de optar na vida. A saúde está diretamente relacionada com as escolhas que não se restringem tão-somente a poder escolher este ou aquele trabalho, realizar-se pessoal e profissionalmente com ele, morar dignamente, comer, relaxar e poder proporcionar condições de vida para os mais próximos, mas também conseguir viver dignamente com base em valores que não predominam em uma sociedade como a brasileira - excludente, individualista, competitiva, consumista. Todos esses são elementos que determinam a nossa saúde que não é só física, mental ou emocional. É tudo junto, ao mesmo tempo! Pensar na saúde do Homem é considerá-lo como ser político - cidadão - e ético - profissional.

Esse entendimento de saúde difere da definição clássica de saúde da Organização Mundial da Saúde - OMS, que relaciona saúde ao completo estado de bem-estar físico, mental e social e pode ser também uma referência para os estudos que têm como foco as atividades físicas como meios para se obter efeitos fisiológicos. Estes podem contribuir para que os sujeitos atinjam bem-estar físico, mental e social. Essa visão de saúde, embora mais ampla que a noção de saúde como meramente ausência de doenças, é considerada por Carvalho (2001) como idealista.
$\mathrm{Na}$ relação entre atividade física de lazer e saúde, observa-se que há diferentes entendimentos, tanto em relação a noção de saúde como do significado da atividade física de lazer. No referencial centrado nas Ciências Biológicas, a atividade física de lazer pode ser compreendida como um meio para os sujeitos atingirem um melhor funcionamento fisiológico do corpo, a fim de alcançarem a sensação de bem-estar ou para se prevenirem de certas doenças. Por outro lado, a partir das Ciências Humanas a atividade física de lazer deve ser um direito dos sujeitos de realizar algo de seu interesse, que pode lhe trazer não somente benefícios fisiológicos e biológicos, mas também benefícios relacionados a cidadania e aos aspectos psicológicos. Estes que pressupõem condições de vida e políticas públicas destinadas a oferta dessas atividades aos sujeitos.

Diante dessas formas distintas de olhares pode-se questionar sobre o que se tem discutido e produzido no meio acadêmico sobre atividade física de lazer e saúde nos últimos seis anos (2010-2015).

\section{Método}

Como estratégia de busca, investigou-se a produção científica relacionada a atividade física de lazer e saúde nos últimos seis anos (2010-2015), tendo como base as orientações para revisão sistemática da literatura de Rother (2007); Costa, Fonseca, Silva Junior e Vasconcelos (2015). A revisão foi feita em bases de dados abrangentes, de trabalhos que tinham um viés tanto das Humanidades como das Ciências Biológicas. Essas bases foram: Scielo e Lilacs e, ainda, uma terceira, os arquivos da Revista Brasileira de Atividade Física \& Saúde, que é uma revista especializada na discussão da atividade física e saúde. As bases de dados foram acessadas em outubro de 2016. A estratégia de busca nas três bases foi a partir do termo "atividade física de lazer e saúde", sendo: Base Lilacs (o termo foi descrito no campo "pesquisa" e em "assunto"); Base Scielo (o termo foi descrito em "busca avançada" [opção "todos os índices" e local "regional"], e no site da Revista Brasileira de Atividade Física \& Saúde (o termo foi descrito no campo "pesquisa" [escopo da busca "todos"]).

Foi feita a opção de busca pelo termo "atividade física de lazer e saúde" e não por palavras em separado, pois na primeira tentativa de uso do termo foi possível identificar 38 artigos. Além disso, o termo contém as principais palavras do presente estudo: atividade física, lazer e saúde. Não foi feita a opção pelo uso dos termos "atividade física relacionada à saúde" + "lazer" porque 
avaliou-se que o uso de um único termo que trouxesse a expressão atividade física de lazer permitiria o acesso a artigos que se destinassem à discussão de um dos conteúdos do lazer (o físicoesportivo), relacionado aos esportes, práticas corporais em geral, pesca e passeios.

Foi feita, ainda, a opção pelo termo atividade física e não outros como, por exemplo, práticas corporais, devido ao fato de a palavra atividade física ser utilizada não só no Brasil, mas também em outros países da América Latina e Caribe, países abrangentes às bases investigadas. É necessário pontuar que o termo "práticas corporais" é frequentemente usado por um grupo de autores brasileiros, que se centram em um referencial das Ciências Humanas, mas seu uso é localizado, pois no exterior não há necessariamente o mesmo entendimento que no Brasil.

Como critérios de inclusão/exclusão, limitamos a busca a anos mais recentes de publicação, recrutamos artigos publicados nos anos de 2010, 2011, 2012, 2013, 2014 e 2015. Não houve restrição de língua em que o texto original estaria escrito. Inicialmente, verificou-se no título dos artigos as palavras atividade física e lazer. Consideramos, ainda, algumas outras palavras dentro do foco da investigação, como "caminhada", por exemplo, pois a entendemos como similar a atividade física. Não houve restrição em relação a população investigada nos estudos. Houve o caso de um mesmo artigo estar em mais de uma base de dados investigada e, nesta situação, mantivemos um artigo para a análise. Os artigos repetidos em mais de uma base de dados foram: Mielke, Malta e Sall (2015); Dias, Loch Ronque(2015); Andrade, Peixoto, Friche, Goston, César, Xavier, Proietti, Roux e Caiaffa (2015); Fernandes, Andrade, Ramos, Friche, Dias, Xavier, Proietti e Caiaffa, (2015); Pegorari, Dias, Santos e Tavares (2015); Marques, Martins, Sarmento, Ramos, Diniz e Costa(2015); Cárdenas-Cárdenas, Burguete-Garcia, Estrada-Velasco, Lopes-Islas, Romero, Cruz e Portillo,(2015); Loch, Porpeta e Brunetto (2015); Santos, Souza, Antes e Dórsil(2015); Kunzler, Rocha, Bombach, Neves, Santos, Carpes (2014); Oliveira, Lopes, Rostile, Werneck, Criep, Deleon e Faerstein (2014); Garcia, Salvador, Sá e Florindo(2014); Monteiro, Neri e Coelim (2014); Boclin, Faerstein e Deleon (2014); Fernandes, Christofaro, Dias, Codogno e Oliveira (2014); Lima, Fermino, Oliveira, Añes e Reis (2013); São-João, Rodrigues, Gallani, Miura, Domingues e Godin (2013); Rocha, Almeida, Araújo, Santos e Rodrigues(2013); Sousa, César, Barros, Carandina,
Goldbaum, Marchioni e Fisberg (2013); Rocha, Almeida, Araújo e Virtuoso(2011a); Rocha, Almeida, Araújo e Virtuoso(2011b); Pitanga e Lessa (2010); Zaitune, Barros, César, Carandina, Goldbaum e Alves (2010); Codogo, Turi, Fernandes e Monteiro(2012); Pazin, Duarte, Borgatto e Peres (2012); Sousa (2012); Inácio, Salvador e Florindo(2011); Canabarro, Neutzling e Rombaldi (2011); Adamoli, Silva e Azevedo(2011); e Lemos, Nakamura, Grisil e Kokubun(2010).

Os artigos disponíveis recentemente na base de dados Lilacs como "Lilacs express" não constavam o período do levantamento bibliográfico, portanto, não foram considerados. Artigos que indicavam no título elementos que não tratavam especificamente da discussão da atividade física de lazer, foram excluídos. A partir de tais critérios para a seleção, os artigos foram lidos e analisados na íntegra. Foram analisados, ao total, 38 artigos. O processo de Revisão Sistemática e a Avaliação dos Artigos encontrados estão no fluxo do processo dos artigos para revisão. Veja a Figura 1 na página seguinte.

Para a avaliação dos artigos utilizou-se uma ficha que continha as categorias: nome do autor; o título do artigo; a principal pergunta a ser respondida na análise "o que se tem discutido e produzido no meio acadêmico sobre atividade física de lazer e saúde nos últimos seis anos (2010-2015).

$\mathrm{Na}$ guia de identificação, são descritos os autores, o periódico, local do estudo, ano de publicação, métodos e técnicas utilizadas em cada pesquisa. Há ainda uma guia de características bibliográficas, com tópicos sobre o ano de publicação, periódico de publicação, países analisados nos estudos, estados e cidades analisados nos estudo e amostra [<500, 500-1500, 1501-6000, >6000].

Também foram analisados "o tipo de atividade física de lazer estudada e o grupo populacional"; "o que se entende por atividade física de lazer?”; “o que se entende por saúde?”

\section{Resultados}

Foi realizada a caracterização bibliográfica dos estudos e notou-se que a produção sobre o tema foi maior no último ano analisado. Dos 38 artigos pesquisados e selecionados, 10 deles foram publicados em 2015, nove em 2014, sete em 2011, Seis em 2013 e 2012 e 2010 com três artigos cada. Apesar de um aumento significativo nos dois últimos anos, a produção também foi expressiva nos anos de 2011 e 2013. No ano de 2012 houve um declínio de publicações com o tema pesquisado. 
FIGURA 1 - Fluxo do processo de seleção dos Artigos para revisão

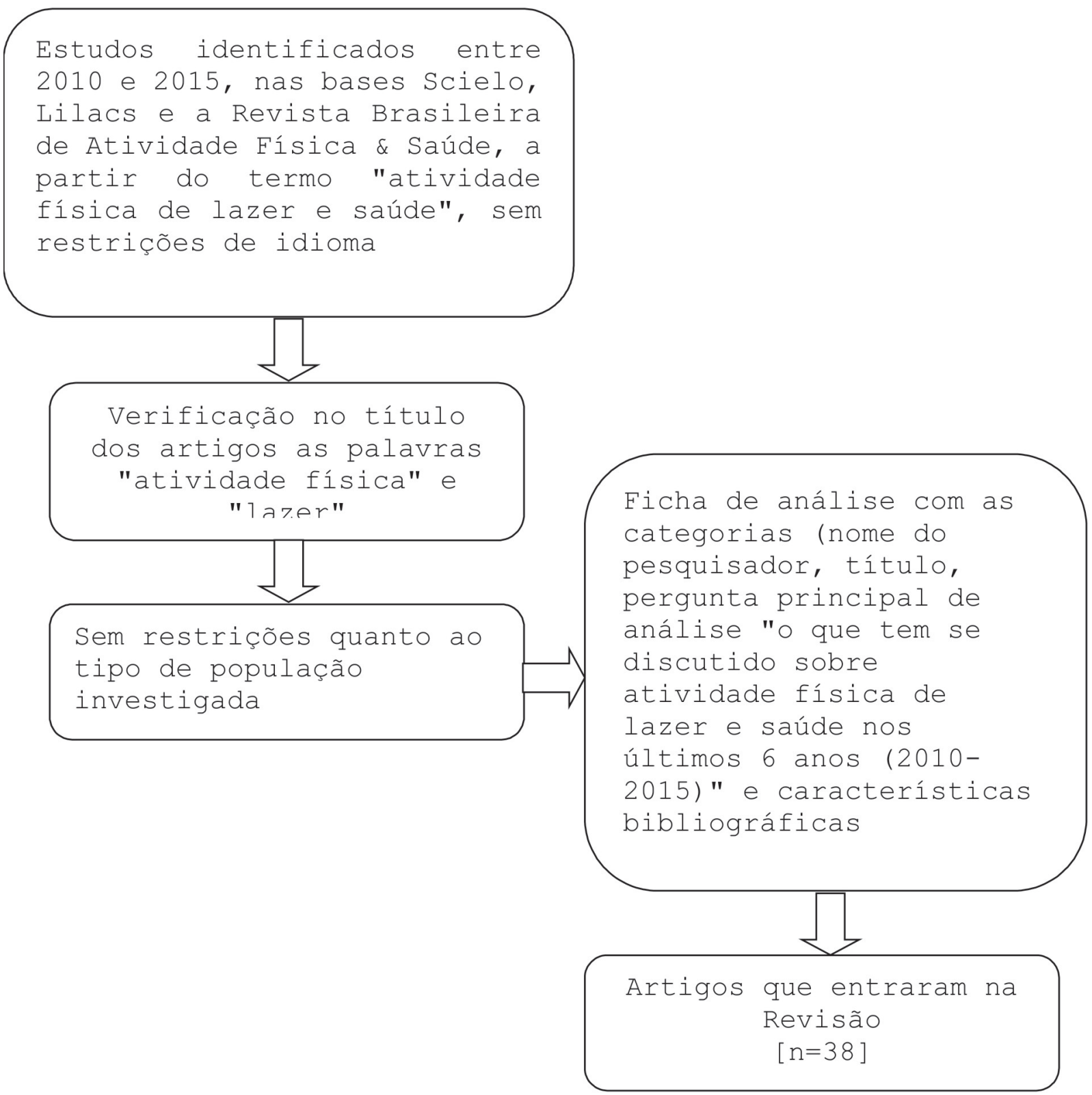

No periódico Revista Brasileira de Atividade Física e Saúde concentrou-se a maioria (12) das publicações sobre o tema. Talvez por ser o único periódico, dentre todos, a tratar especificamente do tema proposto nesta revisão. O segundo foi Cadernos de Saúde Pública com oito artigos nos seis anos analisados.

Em relação à distribuição geográfica destes artigos, a região Sudeste Brasileira concentrou a maior parte dos estudos (16 artigos), seguida pela região Sul (11 artigos) e pela região Nordeste ( 7 artigos). As região Norte e Centro-Oeste do Brasil não apresentaram nenhuma publicação no período analisado. Notou-se que dois estudos foram realizados simultaneamente em várias regiões brasileiras. Duas pesquisas aconteceram em regiões fora do Brasil (um estudo foi realizado no México e outro, em Portugal). Quanto ao método de coletas de dados, verificou-se que o Questionário IPAQ (Questionário Inernacional de Atividades Físicas) foi o mais utilizado, com 13 estudos.

O tipo de estudo predominante foi o quantitativo (30 deles). Os demais estudos (08) com algum tipo de análise qualitativa utilizaram o método quantitativo concomitantemente. Quanto à amostra, sete estudos apresentaram amostras com menos de 500 pessoas. A maior parte dos estudos envolveu entre 501 e 1500 participantes (cerca de 16 estudos) e entre 1501 e 6000 pessoas (cerca 
TABELA 1 - Caracterização bibliográfica dos Artigos

\begin{tabular}{|c|c|}
\hline Ano de Publicação & n \\
\hline 2010 & 3 \\
\hline 2011 & 7 \\
\hline 2012 & 3 \\
\hline 2013 & 6 \\
\hline 2014 & 9 \\
\hline 2015 & 10 \\
\hline Periódico de Publicação & $\mathrm{n}$ \\
\hline Revista Saúde Pública & 2 \\
\hline Rev. Bras. Cineantrop. e Des. Humano & 2 \\
\hline Revista Ciência \& Saúde Coletiva & 4 \\
\hline Revista Escola de Enfermagem & 1 \\
\hline Cadernos de Saúde Pública & 8 \\
\hline Revista Brasileira Medicina do Esporte & 1 \\
\hline Rev. Bras. Atividade Física \& Saúde & 12 \\
\hline Revista Saúde Debate & 1 \\
\hline Sociedade Brasileira de Cardiologia & 1 \\
\hline Jornal Brasileiro de Psiquiatria & 1 \\
\hline Revista Bras. de Ciências do Esporte & 1 \\
\hline Revista Brasileira de Epidemiologia & 2 \\
\hline Journal of Physical Education & 1 \\
\hline Jornal da Pediatria & 1 \\
\hline Pesquisas por Região Estudada & $\mathrm{n}$ \\
\hline Norte Brasileiro $(\mathrm{N})$ & 0 \\
\hline Nordeste Brasileiro (NE) & 7 \\
\hline Centro-oeste Brasileiro (CO) & 0 \\
\hline Sudeste Brasileiro (SE) & 16 \\
\hline Sul Brasileiro (S) & 11 \\
\hline Todas as regiões brasileiras & 2 \\
\hline Regiões fora do Brasil & 2 \\
\hline
\end{tabular}

TABELA 2 - Caracterização metodológica dos Artigos

\begin{tabular}{|c|c|}
\hline Método Coleta de Dados & $\mathrm{n}$ \\
\hline Entrevista e exames antropom./bioquím. & 2 \\
\hline Questionário MLTAQ & 1 \\
\hline Questionário IPAQ & 13 \\
\hline Questionário LTPA & 1 \\
\hline Questionário simples & 9 \\
\hline Questionário BOMFAQ & 1 \\
\hline Entrevistas & 3 \\
\hline Apenas avaliações fís./antrop./bioquím. & 2 \\
\hline Questionário VIGITEL & 1 \\
\hline Questionário GSTPPAQ & 1 \\
\hline Questionário Isaq-A & 1 \\
\hline Formulário SQR-20 & 1 \\
\hline Questionário PAQ-C & 1 \\
\hline Tipo de Estudo & n \\
\hline Quantitativo & 30 \\
\hline Qualitativo & 0 \\
\hline Qualitativo e quantitativo & 8 \\
\hline Por Amostra & $\mathrm{n}$ \\
\hline$<500$ & 7 \\
\hline $501-1500$ & 16 \\
\hline $1501-6000$ & 14 \\
\hline$>6000$ & 1 \\
\hline
\end{tabular}

TABELA 3 - Categorização dos Artigos quanto ao tipo de atividade física e ao conceito de saúde

\begin{tabular}{lc} 
Classificação quanto ao tipo de atividade física & n \\
\hline Atividade física generalizada (não especificada) & 21 \\
Caminhada e outras práticas & 12 \\
Apenas caminhada & 3 \\
Esporte e outras práticas & 2 \\
\hline Classificação quanto ao conceito de saúde & $\mathbf{n}$ \\
\hline Conceito não específico & 7 \\
Conceito saúde (infância /idosos) & 2 \\
Conceito de ausência de doenças & 21 \\
Conceito de autopercepção & 2 \\
Conceito equilíbrio (bio/psico/orgânico/ecológico) & 6 \\
\hline
\end{tabular}


de 14 estudos). Apenas um estudo trabalhou com amostras de mais de 6.000 pessoas.

A maioria dos artigos não especificou a atividade física estudada. Para tais estudos não era significativo a modalidade realizada, mas a prática de qualquer atividade física pelo sujeito investigado. Notou-se, ainda, que a maioria dos pesquisadores se refere às "atividades físicas de lazer" sem, no entanto, se preocupar conceitualmente com o termo "lazer". Quase todos os estudos consideraram a atividade física como aquela atividade realizada obedecendo uma frequência, duração e intensidade dentro dos padrões da saúde, não especificando o que caracteriza a atividade vivenciada no contexto do lazer.

Em relação ao conceito de saúde utilizado pelos pesquisadores, a maioria, apesar de não conceituar diretamente, faz referências à noção de saúde como ausência de doenças.

Notou-se um interesse dos pesquisadores pela população idosa (Zaitune et al., 2010; Inácio, Salvador \& Florindo, 2011; Rocha, Almeida, Araújo \& Virtuoso, 2011b; Mourão, Novais, Andreoni \& Ramos, 2013; Rocha, Almeida, Araújo, Santos \& Rodrigues, 2013; Monteiro, Neri \& Coelim, 2014; Costa, Ribeiro \& Neri, 2015; Pegorari, Dias, Santos \& Tavares, 2015; Santos, Souza, Antes \& Dórsil, 2015), bem como pela classe de professores e estudantes (Canabarro, Neutzling \& Rombaldi, 2011; Sousa \& Nahas, 2011; Pazin, Duarte, Borgatto \& Peres, 2012; Sousa et al., 2013; Silva, Silva \& Spieker, 2014). Houve também interesse por crianças e adolescentes, referenciados em alguns estudos (Lemos, Nakamura, Grisil \& Kokubun, 2010; Lima, Fermino, Oliveira, Añes \& Reis, 2013; Fernandes, Christofaro, Dias, Codogno \& Oliveira, 2014; Rosini, Bruns, Camillo, Machado \& Silva, 2014; Cárdenas-Cárdenas et al., 2015; Dias, Loch \& Ronque, 2015; Fernandes et al., 2015; Loch, Porpeta \& Brunetto, 2015).

Em relação aos problemas estudados, verificamos que as pesquisas podem ser, grosso modo, analisadas em duas frentes: 1) uma, significativamente mais presente, relacionada aos benefícios orgânicos da prática de atividade física, como a pressão arterial, o sono/insônia, o processo de envelhecimento, as doenças, a desnutrição e o uso de medicamentos; 2) outra, encontrada em número bem reduzido, relacionada às questões de cunho mais social e político, como: as preocupações com o transporte público, o apoio social, as instalações de lazer e as políticas de saúde pública e de lazer, de maneira geral.

\section{Discussão}

A principal questão que se tem discutido em relação ao tema atividade física de lazer e saúde é o benefício da atividade física para a saúde. Essas incluem as melhorias com relação ao funcionamento fisiológico do corpo, de modo a evitar doenças ou o uso de medicamentos regularmente. Estes, e outros temas, são exemplos da preocupação central que perpassa os estudos sobre atividade física de lazer e saúde nos últimos seis anos (2010 a 2015).

Em todos os artigos pesquisados, a atividade física de lazer aparece intimamente relacionada à noção de bem-estar. No entanto, a maioria dos artigos analisados não faz jus ao(s) conceito(s) de lazer elaborado(s) pelos autores reconhecidos neste campo de estudo. Como é o caso, por exemplo, do estudo de Codogno, Turi, Fernandes \& Monteiro (2012), que elege como uma de suas preocupações centrais uma demanda por atividades físicas (de lazer) que sejam formalizadas no Sistema Único de Saúde (SUS). Este artigo trata a atividade física de lazer como uma atividade de prescrição médica, tratamento e prevenção da saúde, mas não faz menção a nenhuma outra característica que esteja, de fato, vinculada aos estudos do lazer. De forma semelhante, observou-se artigos que têm como questão central estimar o nível de atividade física de determinada população, bem como a prevalência ou sedentarismo (Lemos, Nakamura, Grisil \& Kokubun, 2010; Pitanga \& Lessa, 2010; Del Duca, Nahas, Hallal \& Peres, 2014; Fernandes Christofaro, Dias, Codogno \& Oliveira, 2014; Garcia, Salvador, Sá \& Florindo, 2014; Rosini, Bruns, Camillo, Machado \& Silva, 2014; Cárdenas-Cárdenas et al., 2015; Costa, Ribeiro \& Neri, 2015; Marques, et al., 2015). Há, ainda, aqueles que apresentam uma preocupação com a população idosa se restringindo ao envelhecimento ativo (Zaitune et al., 2010; Inácio, Salvador \& Florindo, 2011; Rocha, Almeida, Araújo \& Virtuoso, 2011b; Mourão, Novais, Andreoni \& Ramos, 2013; Rocha, Almeida, Araújo, Santos \& Rodrigues, 2013; Monteiro, Neri \& Coelim, 2014; Costa, Ribeiro \& Neri, 2015; Pegorari, Dias, Santos \& Tavares, 2015; Santos, Souza, Antes \& Dórsil, 2015). Fica claro que a atividade física, e não o lazer, é a preocupação central destes estudos.

Verificou-se claramente que o termo lazer aparece, na maioria dos casos, como forma de apresentar o tempo e o local onde as atividades físicas são realizadas. O uso do termo "lazer" oferece uma conotação menos formal à 
prática de atividade física, ampliando, segundo os autores dos artigos, as possibilidades de vivência da atividade física. Ou seja, o termo lazer acompanhando o termo atividade física permite considerar outras práticas que não somente aquelas acompanhadas por profissionais e/ou em contextos "fechados", com o formato de "treinamentos regulares". A forma como cada grupo organiza e realiza a prática de atividade física (frequência, duração e intensidade), bem como os resultados esperados, não parece ter uma relação significativa com as discussões dos estudos do lazer no Brasil e no exterior. Ao contrário, parecem focar somente nos resultados relacionados à saúde e a prevenção de doenças, em sua maioria.

Por outro lado, há alguns artigos (Lima, Fermino, Oliveira, Añes \& Reis, 2013; Mourão, Novais, Andreoni \& Ramos, 2013; Nakamura, Teixeira, Papini, Fernandes \& Kokubun, 2013; Rocha et al., 2013; Boclin, Faerstein \& Deleon, 2014; Oliveira et al., 2014; Silva, Silva \& Spieker, 2014; Dias, Loch \& Ronque, 2015; Fernandes et al., 2015; Pegorari, Dias, Santos \& Tavares, 2015) que se centram em aspectos denominados de "sociais". Esses artigos analisam aspectos como, por exemplo, o de vizinhança - que podem influenciar na regularidade das atividades físicas de lazer - e, ainda, aspectos relacionados as instalações públicas e ao transporte urbano, pois estes podem viabilizar a vivência de atividades físicas de lazer. Há também estudos que envolvem diferenças de desenvolvimentos sociais, tais como nas pesquisas de Costa, Ribeiro e Neri (2015), Andrade et al. (2015), Boclin, Faerstein e Deleon (2014) e Oliveira et al. (2014). Quanto às diferenças de níveis educacionais, os estudos de Mielke, Malta e Sall (2015) e de Marques et al. (2015) também podem ser citados.

Identificou-se, também, diferenças de gêneros nos níveis e na realização das atividades físicas dos estudos. O estudo de Del Duca, Nahas, Hallal e Peres (2014) e Sousa e Nahas (2011), por exemplo, diferenciaram homens e mulheres quanto ao nível de atividade física pela diferença de escolaridade. O estudo de Kunzler, Rocha, Bombach, Neves, Santos e Carpes (2014) diferenciou homens e mulheres pelo tipo de orientação médica quanto à realização das atividades físicas. Já o estudo de Sá Silva, Rodrigues e Salles-Costa (2011), diferenciou as atividades físicas de homens como práticas competitivas, enquanto para mulheres as atividades menos competitivas.

Diante das contribuições tanto das Ciências Biológicas como das Ciências Humanas em relação a discussão das atividades físicas de lazer e saúde, identificou-se na pesquisa realizada que as discussões nos últimos seis anos sobre o tema indicam, em sua maioria, que a relação atividade física de lazer e saúde é compreendida como forma de minimizar ou evitar doenças. O lazer é categoria relacionada ao tempo e local em que os sujeitos realizam a atividade física, não há nos artigos investigados uma maior exploração das contribuições dos estudos do lazer para a saúde da população. Na maioria dos estudos a saúde é compreendida como ausência de doenças.

Esse resultado aponta para a necessidade de uma maior aproximação dos referenciais das Ciências Biológicas e Humanas na intervenção e investigação da atividade física de lazer e suas relações ou contribuição para a promoção da saúde dos sujeitos. Nesse sentido, é fundamental os pesquisadores terem acesso a elementos básicos dos estudos do lazer que poderiam contribuir para ações efetivas nas políticas públicas de saúde, assim como na produção de conhecimento voltada à saúde da população. Alguns desses elementos são, de acordo com Marcellino (2012): as funções do lazer (descanso, diversão e desenvolvimento pessoal e social), a minimização das barreiras sociais do lazer (o que impede os sujeitos de realizarem atividades no contexto do lazer - fator econômico, preconceito, estereótipo etc.), o duplo aspecto educativo do lazer - a educação para e pelo lazer, o acesso dos sujeitos não somente a prática de atividades no contexto do lazer, mas o acesso também ao conhecimento de tais práticas.

As atividades físicas, ao serem consideradas do ponto de vista do lazer, devem ter para os sujeitos um sentido de satisfação pela própria atividade, além de lhes proporcionarem, a partir da mediação do profissional de Educação Física e do lazer, a construção de valores em prol de uma vida melhor. Assim, os sujeitos não devem confundir a prática de atividades físicas no tempo disponível como forma de minimizar ou evitar doenças, que em geral, tem um sentido de obrigação, com as atividades físicas do contexto do lazer. Os sujeitos devem ter opções para poderem fazer escolhas a respeito dessas atividades, de modo a sentirem satisfação na realização das mesmas. Isso é o que caracteriza, de fato, as atividades físicas de lazer em favor da saúde dos sujeitos. Assim, é fundamental que haja a implementação de políticas públicas de esporte e lazer para as populações, de modo que as diferentes práticas relacionadas ao contexto do lazer possam ser oferecidas e garantidas à população, juntamente com o acompanhamento de profissionais e infraestrutura necessária garantida. 


\section{Considerações finais}

Nesse sentido, espera-se com este trabalho contribuir para que outros estudos que enfoquem as atividades físicas de lazer e saúde possam se apoiar nas produções do campo do lazer para potencializarem suas propostas e atender aos problemas investigados, de modo que as pessoas possam, de fato, incluir no seu cotidiano as atividades físicas de lazer, beneficiando-se, não somente do ponto de vista biológico, mas também, social, psicológico, cultural e político.

\section{Referências}

Adamoli, A. N., Silva, M. C. \& Azevedo, M. R. (2012). Prática da caminhada no lazer na população adulta de Pelotas, RS. Rev. Bras. Ativ. Física \& Saúde, 16 (2), 113-119.

Andrade, A. C. de S., Peixoto, S. V., Friche, A. A. de L., Goston, J. L., César, C. C., Xavier, C. C., Proietti, F. A., Roux, A. V. D. \& Caiaffa, W. T. (2015). Social context of neighborhood and socioeconomic status on leisure-time physical activity in a Brazilian urban center: The BH Health Study. Cad. Saúde Pública, 31 (1),136-147.

Boclin, K. L. S., Faerstein, E. \& Deleon, A. C. M. P. (2014). Características contextuais de vizinhança e atividade física de lazer: Estudo Pró-Saúde. Rev. Saúde Pública, 48 (2),249-257.

Canabarro, L. K., Neutzling, M. B. \& Rombaldi, A. J. (2011). Nível de atividade física no lazer dos professores de educação física do ensino básico. Rev. Bras. Ativ. Física \& Saúde, 16(1), 11-17.

Cárdenas-Cárdenas, L. M., Burguete-Garcia, A. I., Estrada-Velasco, B. I., Lopez -Islas, C. L., Romero, J. P., Cruz, M. \& Portillo, M. G. (2015). Leisure-time physical activity and cardiometabolic risk among children and adolescents. Jornal da Pediatria, 91 (2), 136-142.

Carvalho, Y. M. (2001). Atividade física e saúde: onde está e quem é o "sujeito" da relação? Rev. Bras. Ciênc. Esporte, 22 (2), 9-21.

Codogno, J. S., Turi, B., Fernandes \& R., Monteiro, H. (2012). Nível de atividade física no lazer em usuários do sistema único de saúde. Rev. Bras. Ativ. Fís. \& Saúde, 17(6), 543-551.

Costa, M. A. P., Fonseca, M. J. M., Silva Junior, S. H. A. \& Vasconcelos A. G. G. (2015). Características de vizinhança e prática de atividade física: uma revisão sistemática. Rev. Bras. Ativ. Fís. \& Saúde, 20(2), 113-129.

Costa, T. B., Ribeiro, L. H. M. \& Neri, A. L. (2015). Prevalence of and factors associated with leisure-time physical activity in older adults from seven Brazilian cities: data from the FIBRA study. Rev. Bras. Ativ. Física \& Saúde, 20 (2),174-183.

Del Duca, G. F., Nahas, M. V., Hallal, P. C. \& Peres, K. G. (2014). Atividades físicas no lazer entre adultos de Florianópolis, Santa Catarina, Brasil: estudo populacional sobre as características das práticas e de seus praticantes. Ciênc. Saúde Coletiva, 19(11), 4595-4604.

Dias, D. F., Loch, M. R. \& Ronque, E. R. V. (2015). Barreiras percebidas à prática de atividades físicas no lazer e fatores associados em adolescentes. Revista Ciência \& Saúde Coletiva, 20 (11), 3339-3350.

Fernandes, A. P., Andrade, A. C. de S., Ramos, C. G. C., Friche, A. A. de L., Dias, M. A. de S., Xavier, C. C.; Proietti, F. A. \& Caiaffa, W. T. (2015). Atividade física de lazer no território das Academias da Cidade, Belo Horizonte, Minas Gerais, Brasil: o efeito da presença de um programa de promoção da saúde na comunidade. Cad. Saúde Pública, 31 (1), 195-207.

Fernandes, R. A., Christofaro, D. G., Dias, R. M. R., Codogno, J. S. \& Oliveira, A. R. (2014). Desnutrição e sua associação com atividades durante o lazer entre adolescentes. Ciênc. Saúde Coletiva, 19(3), 875-880.

Fraga, A. B. (2012). As práticas corporais e o lazer na contemporaneidade. In Anais do $2^{\circ}$ Colóquio Educação Física e Ciências Sociais em diálogo (pp.28-29). Piracicaba: UNIMEP. Garcia, L. M. T.; Salvador, E. P., Sá, T. H. \& Florindo, A. A. (2014). Association between leisure-time physical activity and long-term medication use in adults from a low socioeconomic region. Rev. Bras. Cineantropom. Desempenho Hum., 16 (4), 371-380.
Inácio, R. F., Salvador, E. P. \& Florindo, A. A. (2011). Análise descritiva da prática de atividade física no lazer de idosos residentes em uma região de baixo nível socioeconômico da zona leste de São Paulo, SP. Rev. Bras. Ativ. Física \& Saúde, 16(2), 150-155.

Isayama, H. F. (2007). Reflexões sobre os conteúdos físico-esportivos e as vivências de lazer. In N. C. Marcellino (Org.), Lazer e cultura (pp.31-46). Campinas, SP: Alínea.

Ministério da Saúde (Brasil). (2012). Glossário temático: promoção da saúde/Ministério da Saúde. Secretaria-Executiva. Secretaria de Vigilância em Saúde. Brasília: Ministério da Saúde.

Kunzler, M. R., Rocha, E. S., Bombach, G. D., Neves, D., Santos, G. S. \& Carpes, F. P. (2014). Saúde no parque: características de praticantes de caminhada em espaços públicos de lazer. Saúde em debate, 38(102), 646-653.

Lemos, N., Nakamura, P. M., Grisil, R. N. F. \& Kokubun, E. (2010). Associação entre nível de atividade física de lazer dos pais com o nível de atividade física dos filhos. Rev. Bras. Ativ. Física \& Saúde, 15(2), 95-100.

Lima, A. V., Fermino, R. C., Oliveira, M. P., Añez, C. R. R. \& Reis, R. S. (2013). Distância percebida até as instalações de lazer e sua associação com a prática de atividade física e de exercícios em adolescentes de Curitiba, Paraná, Brasil. Cad. Saúde Pública, 29(8), 1507-1521.

Marcellino, N. C. (2012). Estudos do laz̧er: uma introdução. 5. ed. Campinas, SP: Autores Associados.

Loch, M. R., Porpeta, R. H. \& Brunetto, B. C. Relação entre a prática de atividade física no lazer dos pais e a dos filhos. (2015). Rev. Bras. Ciênc. Esporte, 37 (1), 29-34.

Marques, A., Martins, J., Sarmento, H., Ramos, M., Diniz, J. \& Costa, F. C. da. Socio-demographic correlates of leisure time physical activity among Portuguese adults. (2015). Cad. Saúde Pública, Rio de Janeiro, 31 (5), 1061-1070.

Mielke, G. I., Malta, D. C. \& Sáll, G. B. A. R. de. Diferenças regionais e fatores associados à prática de atividade física no lazer no Brasil: resultados da Pesquisa Nacional de Saúde-2013. (2015). Rev. Bras. Epidemiologia, 18 (2), 158-169.

Monteiro, N. T., Neri, A. L. \& Coelim, M. F. (2014). Sintomas de insônia, cochilos diurnos e atividades físicas de lazer em idosos: estudo FIBRA Campinas. Rev. Escola Enfermagem USP, 48(2), 242-249.

Mourão, A. R. C., Novais, F. V., Andreoni, S. \& Ramos, L. R. (2013). Atividade física de idosos relacionada ao transporte e lazer, Maceió, Brasil. Rev. de Saúde Pública, 47(6), 1112-1122.

Nakamura, P. M., Teixeira, I. P., Papini, C. B., Fernandes, R. A. \& Kokubun, E. (2013). Associação da caminhada no lazer e no transporte com ambiente construído em adultos do município de Rio Claro - SP. Rev. Bras. Ativ. Fís. ¿ Saúde, 18(4), 424-434.

Oliveira, A. J., Lopes, C. S., Rostila, M., Werneck, G. L., Criep, R. H., Deleon, A. C. M. P. \& Faerstein, E. (2014). Gender differences in social support and leisure-time physical activity. Rev. Saúde Pública, 48(4), 602-612.

Pazin, J., Duarte, M. F. S., Borgatto, A. F. \& Peres, M. A. (2012). Ambiente urbano percebido e apoio social para a atividade física no lazer e no deslocamento em adultos de Florianópolis, SC. Rev. Bras. Ativ. Fís. \& Saúde, 17(2), 100-106

Pegorari, M. S., Dias, F. A., Santos, N. M. de F. \& Tavares, D. M. dos S. (2015). Prática de atividade física no lazer entre idosos de área rural: condições de saúde e qualidade de vida. Journal of Physical Education, 26 (2), 233-241.

Pitanga, F. J. G. \& Lessa, I. (2010). Associação entre atividade física no tempo livre e pressão arterial em adultos. Arq. Bras. Cardiologia, 95(4),480-485.

Rocha, S. V., Almeida, M. M. G., Araújo, T. M., Santos, L. B. \& Rodrigues, W. K. M. (2013). Fatores associados à atividade física insuficiente no lazer entre idosos. Rev. Bras. Med. Esporte, 19(3), 191-195.

Rocha, S. V., Almeida, M. M. G., Araújo, T. M. \& Virtuoso Junior, J. S. (2011a). Fatores associados à atividade física no lazer entre residentes de áreas urbanas de um município do nordeste do Brasil. Rev. Bras. Cineantropom. Desemp Hum., 2011, 13(4), 257-264.

Rocha, S. V., Almeida, M. M. G., Araújo, T. M. \& Virtuoso Junior, J. S. (2011b). Atividade física no lazer e transtornos mentais comuns entre idosos residentes em um município do Nordeste do Brasil. J. Bras Psiquiatr, 60(2), 80-85.

Rosini, N., Bruns, E., Camillo, G., Machado, M. \& Silva, E. (2014). Jogos recreativos melhoram os fatores de risco cardiovascular em crianças e adolescentes com dislipidemia e obesidade abdominal: um estudo piloto. Rev. Bras. de Ativ. Fís. \& Saúde, 19(1), 121-132. 
Rother, E. T. (2007). Revisão sistemática x revisão narrativa. Acta Paulista de Enfermagem, 20(2), V-VI.

Sá Silva, S. O., Sandre-Pereira, G. \& Salles-Costa, R. (2011). Fatores sociodemográficos e atividade física de lazer entre homens e mulheres de Duque de Caxias-RJ. Ciênc. Saúde Coletiva, 16 (11), 4491-4501.

São-João, T. M., Rodrigues, R. C. M., Gallani, M. C. B. J., Miura, C. T. P., Domingues, G. L. D. \& Godin, G. (2013). Adaptação cultural da versão brasileira do Godin-Shephard Leisure-Time Physical Activity Questionnaire. Rev. de Saúde Pública, 47(3), 479-487.

Santos, F. A. A., Souza, J. B., Antes, D. L. \& Dórsil, E. (2015). Prevalência de dor crônica e sua associação com a situação sociodemográfica e atividade física no lazer em idosos de Florianópolis, Santa Catarina: estudo de base populacional. Rev. Bras. Epidemiologia, 18 (1), 234-247.

Silva, M., Silva, L. \& Spieker, C. (2014). Atividade física no lazer e fatores associados em professores pré-escolares de Pelotas - RS, Brasil. Rev. Bras. de Ativ. Fís. \& Saúde, 19(4), 417:428.
Sousa, C. A., Cesar, C. L. G., Barros, M. B. A.; Carandina, L., Goldbaum, M., Marchioni, D. M. L. \& Fisberg, R. M. (2013). Prevalência de atividade física no lazer e fatores associados: estudo de base populacional em São Paulo, Brasil, 2008-2009. Cad. Saúde Pública, 29(2), 270-282.

Sousa, T. F. (2012). Atividades físicas praticadas no lazer por universitários de uma instituição pública do Nordeste do Brasil. Rev. Bras. Ativ. Física ¿. Saúde, 17(2), 125-131.

Sousa, T. F. \& Nahas, M. V. (2011). Prevalência e fatores associados a menores níveis de prática de atividades físicas no lazer em estudantes de uma universidade pública do Estado da Bahia. Rev. Bras. Ativ. Física \& Saúde, 16(4), 322-329.

Zaitune, M. P. A., Barros, M. B. A., César, C. L. G., Carandina, L., Goldbaum, M. \& Alves, M. C. G. P. (2010). Fatores associados à prática de atividade física global e de lazer em idosos: Inquérito de Saúde no Estado de São Paulo (ISA-SP), Brasil. Cad. Saúde Pública, 26 (8), 1606-1618.

Submetido em: 26-12-2016

Aceito em: 20-6-2017 